\title{
Vibration Analysis Of a Self-Excited Elastic Beam
}

\author{
M. A. Barrón-Meza \\ Departamento de Materiales \\ Universidad Autónoma Metropolitana Azcapotzalco \\ Av. San Pablo 180, Col. Reynosa-Tamaulipas \\ C.P. 02200, México, D.F., MÉXICO \\ bmma@correo.azc.uam.mx
}

\begin{abstract}
The vibration behavior and the energy exchange among the normal modes of a clamped-free self-excited elastic beam are analyzed in this work. To model this kind of beam, the damping term of a van der Pol oscillator is directly added to the equation of a linear elastic beam, yielding a single nonlinear partial differential equation. To solve this equation, a spectral method is employed. Three vibration modes are considered in the analysis, and the values of the self-exciting constant are varied in order to cover from linear to nonlinear vibration behavior. Multiple frequencies of the nonlinear beam are determined through the power spectral density of the beam free-end time series. Given that this relatively simple model mimics at least in a qualitative way some key issues of the fluid-structure problem, it could be potentially useful for fatigue studies and vibration analysis of rotating blades in turbomachinery.
\end{abstract}

Keywords: Beam vibration, fluid-structure problem, modal interaction, self-excited beam, spectral method, turbine blade vibration, van der Pol oscillator.

\section{RESUMEN}

En este trabajo se analizan el comportamiento bajo vibración y el intercambio de energía entre los modos normales de una barra elástica autoexcitada con un extremo fijo y el otro libre. Para modelar esta clase de barra se le agrega directamente el término de amortiguamiento de un oscilador van der Pol a la ecuación de una barra elástica lineal, obteniéndose una sola ecuación diferencial parcial. Para resolver esta ecuación se usa el método espectral. En el análisis se consideran tres modos de vibración, y los valores de la constante de auto-excitación se varían a modo de cubrir un comportamiento a la vibración desde lineal hasta no lineal. Las múltiples frecuencias de la barra no lineal se determinan mediante el espectro de potencias de las series de tiempo del extremo libre. Dado que este modelo relativamente simple reproduce, al menos cualitativamente, algunos aspectos clave del problema fluido-estructura, puede ser potencialmente útil para estudios de fatiga y análisis de la vibración de álabes rotatorios en turbomaquinaria.

\section{Introduction}

Vibration of turbine blades in turbomachinery may cause failure by fatigue $[1,2]$. Experimental analysis of blade vibration is very difficult because sensors are intrusive and interfere with machine operation. Also, noncontact measurement techniques have been proposed; however, frequently, they are expensive [3]. On the other hand, numerical studies of blade vibration require a lot of computational power given that the fluidstructure problem must be solved [4-6]. From a formal point of view, the fluid-structure problem requires the numerical solution of the NavierStokes equations coupled with the equations of elasticity for the structure using Direct Numerical Simulation and Finite Element techniques, respectively; however, both approaches can be very expensive from a computational point of view $[7,8]$. Then, a simplified model which simulates the fluid-structure interaction of a turbine blade, but at the same time not be very computationally demanding, is required.

Frequently turbine blades are represented in the literature as elastic clamped-free beams, both in numerical [9-14] and experimental [15] studies. Self-excitation can be taken into account by introducing a nonlinear damping term in the classical linear beam equation to simulate the fluidstructure interaction in turbine blades [13]. This is justified by fact that the natural frequency of the self-excited oscillator is proportional to the free stream velocity and the structure motion interacts 
with the oscillator [16]. The van der Pol oscillator is one that has been used in the literature: in [17] it is suggested that a van der Pol type oscillator can be used to represent the time-varying forces on a cylinder due to vortex shedding. In [18] this oscillator is used for the study of an aeroelastic system possessing limit cycle oscillations. Also, in [19] a van der Pol-based model that captures many of the features seen in experimental results of an elastically mounted cylinder exposed to wind is introduced. Sometimes, self-excitation in fluidstructure problems is considered by introducing an additional van der Pol equation, which is named in the literature as a wake-oscillator model $[16,20]$. Both the beam equation and the wake-oscillator model are coupled through the acceleration term and they are simultaneously solved using numerical techniques, i.e. finite differences [6] and collocation [21]. Unfortunately, wake-oscillator models are best suited for long flexible slender structures exposed to vortex induced vibration, and therefore they have been mainly applied in the ocean engineering field in structures such as mooring cables and oil risers $[6,22]$.

In this work the transient and long-term vibration behavior and the energy exchange among modes of a clamped-free self-excited elastic beam are analyzed. A van der Pol self-exciting term is directly added to the equation of a linear beam to represent through a single partial differential equation the fluid-structure interaction problem and model nonlinearities of a turbine blade. The parameter that indicates the strength of the damping is the self-excitation constant, A. By means of the spectral method, the partial differential equation is transformed into a set of coupled nonlinear ordinary differential equations which are numerically solved. Given that high vibration amplitudes associated to low frequencies are significant for failure by fatigue [23], to simplify the computational task and the analysis of the modal interaction, just three vibration modes are considered. Values of $A$ are varied from 0 to 10 in order to study from linear to nonlinear the vibration behavior of the self-excited beam. For $A \in[0,0.33]$, a single vibration frequency is present and the beam remains vibrating in the initially excited mode, i.e. no energy transfer between modes arises and the beam behaves in a linear fashion. For $A>0.33$ multiple frequencies are present and the vibration behavior is nonlinear. In this case the power spectral density based on the backward Discrete Fourier Transform of the beam free-end time series is employed to determine the dominant frequency.

\section{Mathematical model}

Formally, tackling the fluid-structure problem requires the numerical solution of the NavierStokes equations [6-8]. This procedure results expensive from a computational point of view; therefore, new less computationally expensive models to study fluid-structure interaction in turbine blade and elastic beam vibration problems are needed.

To simplify the vibration analysis, turbine blades are frequently modeled in the literature as elastic clamped-free beams in numerical [9-14] and experimental [15] studies. The required fluidstructure interaction is taken into account by introducing a self-exciting function, such as the given by the van der Pol oscillator, in the classical linear beam equation. This is justified by fact that the natural frequency of the self-excited oscillator is proportional to the free stream velocity and the structure motion interacts with the oscillator [16]. Besides, a van der Pol type oscillator can be used to represent the time-varying forces on a cylinder due to vortex shedding [17].

The transverse motion of an elastic beam of homogeneous section and properties is governed by Equation [24]:

$$
\frac{\partial^{4} y}{\partial x^{4}}+\frac{1}{a^{2}} \frac{\partial^{2} y}{\partial t^{2}}=0
$$

with

$$
\mathrm{a}^{2}=\frac{\mathrm{EI}}{\rho \mathrm{A}_{\mathrm{c}}}
$$

where $x$ is the distance from the root, $y(x, t)$ is the transverse displacement, $t$ is time, and $E, I, \rho$ and $A_{c}$ are the Young's modulus, the moment of inertia, the mass density per unit volume, and the cross sectional area of the beam, respectively. Defining the non-dimensional variables $X=x / L, Y=y / L, \tau=$ 
ta/ $L^{2}$, where $L$ is the beam length, the following is obtained:

$$
\frac{\partial^{4} Y}{\partial X^{4}}+\frac{\partial^{2} Y}{\partial \tau^{2}}=0
$$

The van der Pol equation is a well known second order ordinary differential equation used in 1928 by B. van der Pol and J. van der Mark $[25,26]$ to represent, by first time, non-electrical nonlinear oscillators. As time has elapsed, it has been widely used in many disciplines to model any kind of relaxation oscillator. In this work the following version of the van der Pol equation is proposed:

$$
\frac{\partial^{2} Y}{\partial \tau^{2}}+A\left(Y^{2}-1\right) \frac{\partial Y}{\partial \tau}+Y=0
$$

where $A$ is the self-exciting constant, which is a parameter that measures the strength of the nonlinear damping [27]. Besides, self-sustained oscillations are provided by the term $\mathrm{Y}^{2}-1$.

In this work the nonlinear term $A\left(Y^{2}-1\right)$ of the van der Pol equation is directly added into the linear non-dimensional beam equation to model the selfsustained oscillations and include the fluidstructure interaction. As stated above, this is justified by the fact that the van der Pol oscillator has been used in the literature for a long time to represent the time-varying forces on a cylinder due to vortex shedding $[17,19]$. Then, by adding the nonlinear damping and the self-sustaining terms of Eq. (4) into Eq. (3), a single partial differential equation arises:

$$
\frac{\partial^{4} \mathrm{Y}}{\partial \mathrm{X}^{4}}+\frac{\partial^{2} \mathrm{Y}}{\partial \tau^{2}}+\mathrm{A}\left(\mathrm{Y}^{2}-1\right) \frac{\partial \mathrm{Y}}{\partial \tau}=0
$$

Eq. (5) supposedly retains two key issues of the fluid-structure problem: the nonlinear fluid-structure interaction and the elastic response of the structure. These issues are responsible of the complex vibration behavior of rotating blades in turbomachinery.

\section{Boundary conditions}

A clamped-free beam, self-excited or not, is a beam that is only supported on one of its ends while the other end is free. Mathematically, for this kind of beam the boundary conditions are as follows [24]. For the clamped end, $X=0$ :

$$
\begin{aligned}
& Y(0)=0 \\
& \frac{\partial Y(0)}{\partial X}=0
\end{aligned}
$$

and for the free end, $\mathrm{X}=\mathrm{L}$ :

$$
\frac{\partial^{2} \mathrm{Y}(\mathrm{L})}{\partial \mathrm{X}^{2}}=0
$$

$$
\frac{\partial^{3} \mathrm{Y}(\mathrm{L})}{\partial \mathrm{X}^{3}}=0
$$

\section{Spectral solution}

To determine the natural frequencies and normal vibration modes of the self-excited beam, the spectral method is employed. This method is based on the decomposition of a partial differential equation into an infinite summation of two series of orthogonal functions [28]. Then, the dimensionless transverse displacement $Y(\tau, X)$ of the beam can be expressed in terms of the natural vibration modes as [29]

$$
\mathrm{Y}(\tau, \mathrm{X})=\sum_{\mathrm{i}=1}^{\infty} \mathrm{a}_{\mathrm{i}}(\tau) \Phi_{\mathrm{i}}(\mathrm{X})
$$

where $\mathrm{a}_{\mathrm{i}}(\tau)$ is a time dependent coefficient and $\Phi_{i}(X)$ is the ith normal vibration mode of the beam. The assumption of orthogonality requires that [24]

$$
\left\langle\Phi_{\mathrm{i}}, \Phi_{\mathrm{j}}\right\rangle=\int_{0}^{\mathrm{L}} \Phi_{\mathrm{i}} \Phi_{\mathrm{j}} \mathrm{dx}=0
$$

for $i \neq j$. 


\section{Vibration Analysis Of a Self-Excited Elastic Beam, M. A. Barrón-Meza, 227-239}

A solution for $\Phi \mathrm{i}(\mathrm{X})$ of Eq.(10) for the nonlinear beam in terms of a combination of sines, cosines, hyperbolic sines and hyperbolic cosines functions can be assumed of the form [24]

$\Phi_{i}(X)=C_{i 1} \sin \left(\beta_{i} X\right)+C_{i 2} \cos \left(\beta_{i} X\right)+C_{i 3} \sinh \left(\beta_{i} X\right)+C_{i 4} \cosh \left(\beta_{i} X\right)$

where $\beta_{i}$ is the eigenvalue corresponding to the ith mode, and $C_{i 1}, C_{i 2}, C_{i 3}$ and $C_{i 4}$ are constants associated to the ith mode, whose values are determined from the boundary conditions. Moreover, it can be verified that Eq. (12) satisfies the ortoghonality condition given by Eq. (11) [24]. The following relationship arises when Eq. (12) is four times derived respect to $X$ :

$$
\Phi_{\mathrm{i}}^{\mathrm{m} ' \mathrm{X}}(\mathrm{X})=\beta_{\mathrm{i}}^{4} \Phi_{\mathrm{i}}(\mathrm{X})
$$

where $\Phi i^{\prime \prime \prime ' ~ i s ~ t h e ~ f o u r t h ~ d e r i v a t i v e ~ o f ~} \Phi_{i}$ respect to X.Substituting Eq. (10) into Eq. (5) gives

$$
\sum_{\mathrm{i}=1}^{\mathrm{n}} \mathrm{a}_{\mathrm{i}} \Phi_{\mathrm{i}}^{\mathrm{\prime \prime \prime}}+\sum_{\mathrm{i}=1}^{\mathrm{n}} \mathrm{a}_{\mathrm{i}}^{\prime \prime} \Phi_{\mathrm{i}}+\mathrm{A}\left(\left(\sum_{\mathrm{i}=1}^{\mathrm{n}} \mathrm{a}_{\mathrm{i}} \Phi_{\mathrm{i}}\right)^{2}-1\right) \sum_{\mathrm{i}=1}^{\mathrm{n}} \mathrm{a}_{\mathrm{i}}^{\prime} \Phi_{\mathrm{i}}=0
$$

where $a_{i}$ and $a_{i}$ " are the first and the second time derivatives of $a_{i}$, respectively, and $n$ is the number of vibration modes considered. Expanding and rearranging the above equation yields

$$
\sum_{i=1}^{n} a_{i}^{\prime \prime} \Phi_{i}+A\left(\sum_{i=1}^{n} a_{i}^{2} \Phi_{i}^{2}+\sum_{i=1}^{n} a_{i} \Phi_{i} \sum_{k=1 \neq i}^{n} a_{k} \Phi_{k}-1\right) \sum_{i=1}^{n} a_{i}^{\prime} \Phi_{i}+\sum_{i=1}^{n} a_{i} \Phi_{i}^{\prime \prime \prime \prime}=0
$$

Substituting Eq. (13) into Eq. (15), taking inner product against $\Phi_{\mathrm{j}}$ and rearranging it is obtained:

$$
\left(\mathrm{A}_{\mathrm{i} 1}\right) \mathrm{a}_{\mathrm{i}}^{\prime \prime}+\left(\mathrm{A}_{\mathrm{i} 2}\right) \mathrm{a}_{\mathrm{i}}^{\prime}+\left(\mathrm{A}_{\mathrm{i} 3}\right) \mathrm{a}_{\mathrm{i}}+\mathrm{f}_{\mathrm{i}}=0
$$

Eq. (16) represents a set of $n$ coupled second order nonlinear ordinary differential equations. Coefficients of such equations are given by

$$
\begin{gathered}
\mathrm{A}_{\mathrm{i} 1}=\left\langle\Phi_{\mathrm{i}}, \Phi_{\mathrm{i}}\right\rangle \\
\mathrm{A}_{\mathrm{i} 2}=\mathrm{A}\left(\sum_{\mathrm{i}=1}^{\mathrm{n}} \mathrm{a}_{\mathrm{j}}^{2}\left\langle\Phi_{\mathrm{i}} \Phi_{\mathrm{j}}^{2}, \Phi_{\mathrm{i}}\right\rangle+2 \sum_{\mathrm{i}=1}^{\mathrm{n}-1} \sum_{\mathrm{k}=\mathrm{i}+1}^{\mathrm{n}} \mathrm{a}_{\mathrm{j}} \mathrm{a}_{\mathrm{k}}\left\langle\Phi_{\mathrm{i}} \Phi_{\mathrm{j}} \Phi_{\mathrm{k}}, \Phi_{\mathrm{i}}\right\rangle-\left\langle\Phi_{\mathrm{i}}, \Phi_{\mathrm{i}}\right\rangle\right)
\end{gathered}
$$




$$
\begin{gathered}
\mathrm{A}_{\mathrm{i} 3}=\beta_{\mathrm{i}}^{4}\left\langle\Phi_{\mathrm{i}}, \Phi_{\mathrm{i}}\right\rangle \\
\mathrm{f}_{\mathrm{i}}=\mathrm{A} \sum_{\mathrm{m}=\mathrm{l} \neq \mathrm{i}}^{\mathrm{n}} \mathrm{a}_{\mathrm{m}}^{\prime}\left(\sum_{\mathrm{j}=1}^{\mathrm{n}} \mathrm{a}_{\mathrm{j}}^{2}\left\langle\Phi_{\mathrm{m}} \Phi_{\mathrm{j}}^{2}, \Phi_{\mathrm{i}}\right\rangle+2 \sum_{\mathrm{j}=1}^{\mathrm{n}-1} \sum_{\mathrm{k}=\mathrm{j}+1}^{\mathrm{n}} \mathrm{a}_{\mathrm{j}} \mathrm{a}_{\mathrm{k}}\left\langle\Phi_{\mathrm{m}} \Phi_{\mathrm{j}} \Phi_{\mathrm{k}}, \Phi_{\mathrm{i}}\right\rangle\right)
\end{gathered}
$$

\section{Eigenvalues and eigenfrequencies}

Eigenvalues $\beta_{i}$ corresponding to the ith mode can be determined by numerically solving the following expression [24]

$$
\cos \left(\beta_{\mathrm{i}} \mathrm{L}\right)+\cosh \left(\beta_{\mathrm{i}} \mathrm{L}\right)+1=0
$$

and the angular eigenfrequencies $\omega_{i}$ associated to the corresponding eigenvalues are estimated from [24]

$$
\omega_{\mathrm{i}}=\beta_{\mathrm{i}}^{2}
$$

Substituting Eq. (12) into the boundary conditions in $X=0$ given by Eqs. (6-7) gives

$$
\begin{aligned}
& \mathrm{C}_{\mathrm{i} 3}=-\mathrm{C}_{\mathrm{i} 1} \\
& \mathrm{C}_{\mathrm{i} 4}=-\mathrm{C}_{\mathrm{i} 2}
\end{aligned}
$$

Considering the boundary conditions in $\mathrm{X}=\mathrm{L}$ defined by Eqs. (8-9), the following expression for $\mathrm{C}_{\mathrm{i} 2}$ is obtained

$$
\mathrm{C}_{\mathrm{i} 2}=-\mathrm{C}_{\mathrm{i} 1}\left(1+\mathrm{v}_{\mathrm{i}}\right)
$$

where

$$
v_{i}=\frac{e^{-\beta_{i} \mathrm{~L}}+\sin \left(\beta_{\mathrm{i}} \mathrm{L}\right)+\cos \left(\beta_{\mathrm{i}} \mathrm{L}\right)}{\sinh \left(\beta_{\mathrm{i}} \mathrm{L}\right)-\sin \left(\beta_{\mathrm{i}} \mathrm{L}\right)}
$$

\section{Results and discussion}

To simplify the computational task and the analysis of the modal interaction, just three vibration modes are considered in Eq. (10). From a more practical point of view, this is justified by the fact that the first modes have low frequencies and high vibration amplitudes, factors which are significant in failure by fatigue of turbine blades [23].

Eq. (21) is numerically solved for the eigenvalues $\beta_{i}$ using the first order Newton-Raphson procedure. Eigenfrequencies $\omega_{i}$ associated to the eigenvalues are determined from Eq. (22). Table 1 shows the values of the eigenvalues and their corresponding eigenfrequencies for the first three vibration modes of the self-excited beam assuming the dimensionless value $L=1$. By fixing $C_{i 1}=1$ and calculating $\mathrm{C}_{\mathrm{i} 2}$ from Eqs. (25) and (26), values of $\mathrm{C}_{\mathrm{i} 3}$ and $\mathrm{C}_{\mathrm{i} 4}$ are easily determined from Eqs. (23) and (24). In Table 2 the values of the above constants are shown. The three natural vibration modes are calculated from Eq. (12) using the values of $\beta_{i}$ and $C_{i}$ from Tables 1 and Table 2, respectively. The time dependent coefficients $a_{i}$ of Eq. (10) are obtained by numerically solving Eq. (16) through the classical Fourth Order RungeKutta method using a dimensionless time step of $\Delta \tau=10^{-6}$.

\begin{tabular}{|c|c|c|}
\hline ith Mode & $\mathrm{B}_{\mathrm{i}}$ & $\mathrm{F}_{\mathrm{i}}, \mathrm{Hz}$ \\
\hline 1 & 1.8751 & 0.5596 \\
\hline 2 & 4.6941 & 3.5063 \\
\hline 3 & 7.8548 & 9.8232 \\
\hline
\end{tabular}

Table 1. Eigenvalues and eigenfrequencies of the considered vibration modes. 


\begin{tabular}{|c|c|c|c|c|}
\hline ith Mode & $\mathrm{C}_{\mathrm{i} 1}$ & $\mathrm{C}_{\mathrm{i} 2}$ & $\mathrm{C}_{\mathrm{i} 3}$ & $\mathrm{C}_{\mathrm{i} 4}$ \\
\hline 1 & 1.0000 & -1.3622 & -1.0000 & 1.3622 \\
\hline 2 & 1.0000 & -0.9819 & -1.0000 & 0.9819 \\
\hline 3 & 1.0000 & -1.0008 & -1.0000 & 1.0008 \\
\hline
\end{tabular}

Table 2. Numerical values of the constants of Eq. (12).

Once $a_{i}(\tau)$ and $\Phi_{i}(X)$ are known, the beam dimensionless transverse displacement $Y(\tau, X)$ is determined from Eq. (10). A dimensionless integration time of 1000 is considered for the longterm runs given that the numerical simulations showed that in this time the vibration pattern of the beam remains stable. A tracking point is located at the free-end of the beam corresponding to $X=L=1$. Through a power spectral density analysis based on the backward Discrete Fourier Transform, singles or multiple frequencies of the free-end time series are determined.

\subsection{Linear beam}

For $A=0$, one has the classical and well-known linear beam whose vibration modes are uncoupled and no energy transfer is present among natural vibration modes. The self-excited beam remains vibrating, in transient or steady state, in the mode initially excited. For $A €[0,0.27]$, the self-excited beam still behaves as a linear one; however, for
$A €[028,0.33]$, some energy transfer among modes is observed when the first mode is excited. This transfer is demonstrated through the presence of two frequencies of the free-end time series of Fig. 1 for $A=0.28$, where it can be appreciated that the beam vibration consists of a high frequency component riding over a low frequency component. Besides, Fig. 1 shows a strongly modulated interaction between widely spaced modes, i.e. the first and the third modes. This behavior has been reported in actual beams with external excitation [23]. The dominance of a frequency of $0.56 \mathrm{~Hz}$ belonging to the first mode becomes evident in the power spectrum of the above time series shown in Fig. 2, where a small peak of $9.82 \mathrm{~Hz}$, which corresponds to the third vibration mode, is present. This kind of power spectrum is reported in actual steam turbine blades [2]. In spite of the presence of an additional frequency, for the self-excited beam the long-term dominant frequency corresponds to the initially excited mode, i.e. the first one.

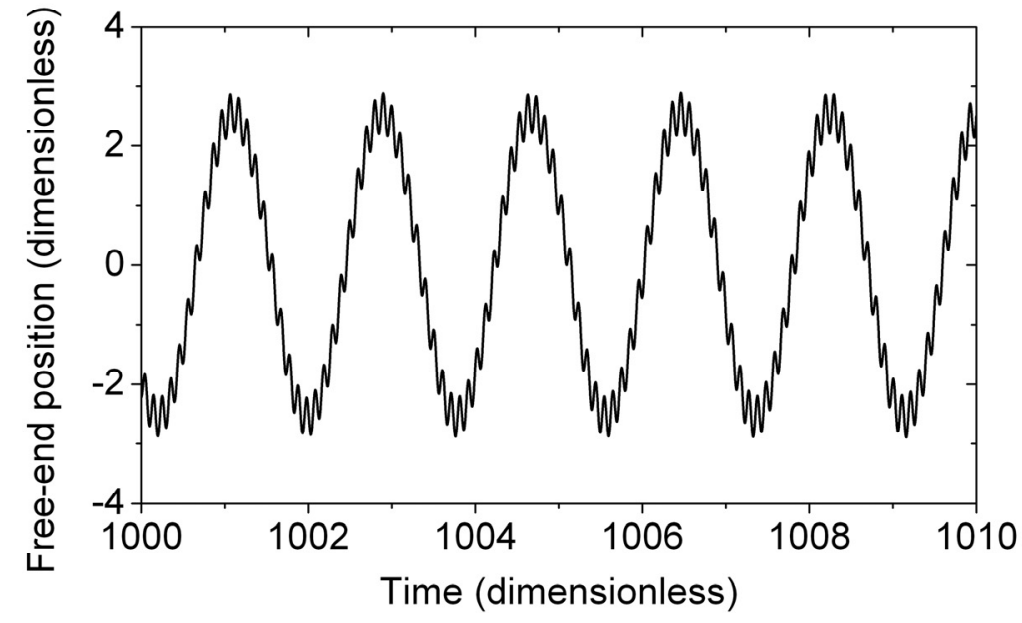

Figure 1 . Time series for $A=0.28$. The first mode is initially excited. 


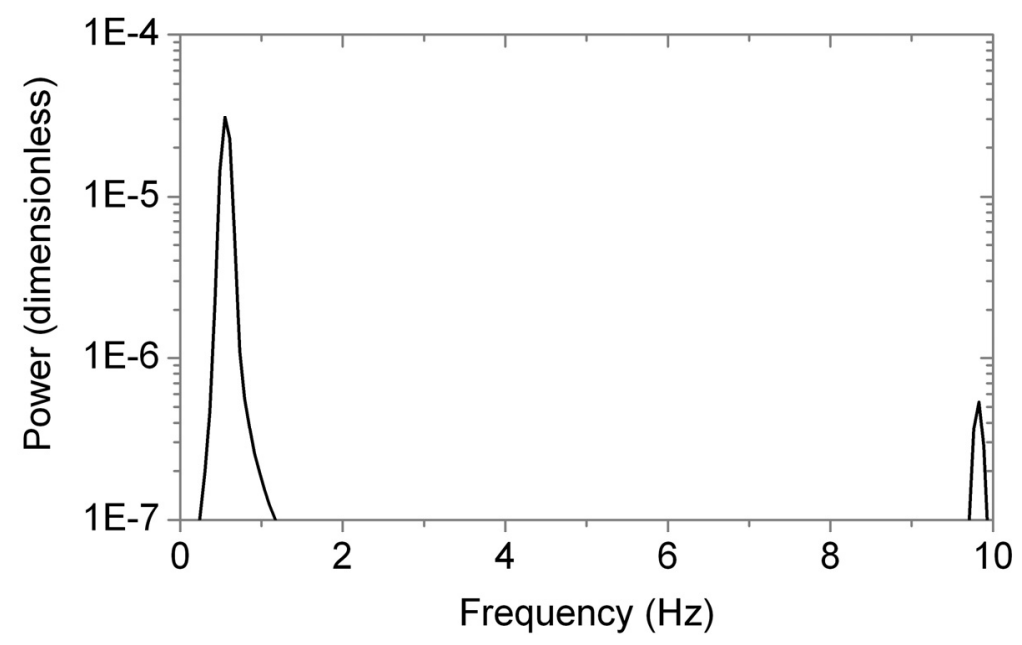

Figure 2. Power spectrum corresponding to the time series of Fig. 1.

When the second or the third modes are excited for $A €[0.28,0.33]$, the self-excited beam remains vibrating in the corresponding mode, and therefore the beam behaves in a linear fashion.

\subsection{Nonlinear beam}

Transfer of energy among vibration modes is characteristic of nonlinear beams [23]. For values of the self-exciting constant beyond a critical value, the considered nonlinear self-excited beam vibrates in a complex way. This behavior becomes apparent in the form of vibration attractors with multiple frequencies. Numerical results show that the dominant frequency of a particular attractor depends on the value of the self-exciting constant and on the initially excited mode. Due to the energy transfer among modes, the long-term dominant frequency does not always correspond to the mode initially excited.

In Fig. 3 the dominant frequencies of the selfexcited beam at long times for $A €[0,10]$ when each vibration mode is separately excited are shown. Similarly to the case of small values of the selfexciting constant, Fig. 3 shows that the nonlinear self-excited beam resembles a linear one when the second and the third modes are individually excited. On the contrary, a rich nonlinear long-term behavior is exhibited by the self-excited beam when just the first mode is excited. The following results and discussion correspond to this case.

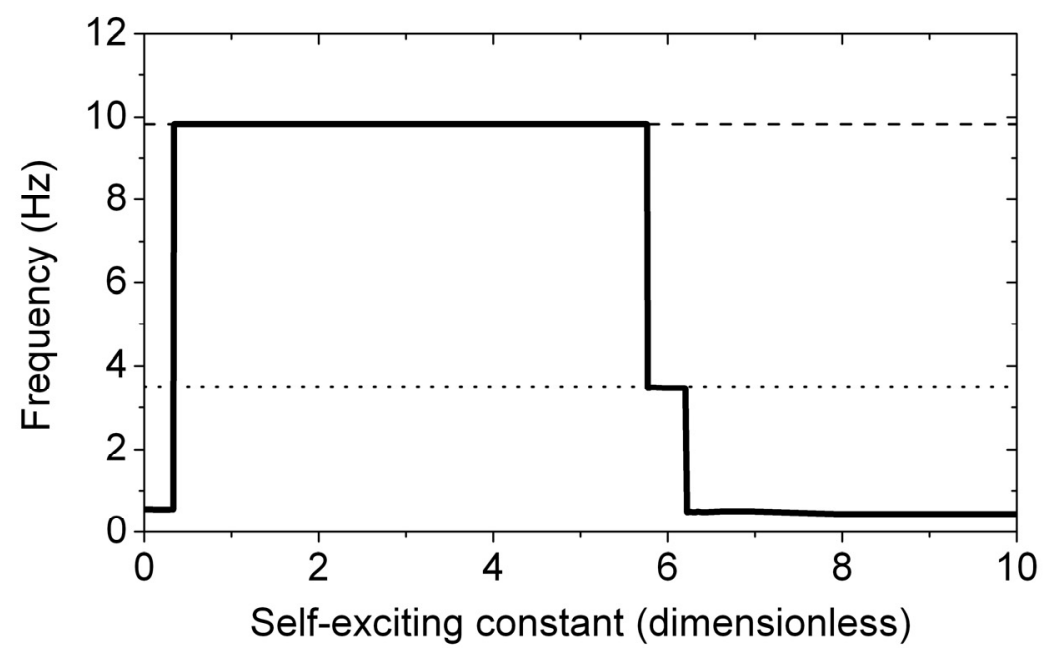

Figure 3. Beam dominant frequencies for long times as a function of the self-exciting constant. Excited vibration modes: thick line, first mode; dotted line, second mode; dashed line, third mode. 
For $A €[0.34,5.76]$, energy is transferred from the first mode to the third one, and the attractor has a single frequency of $9.82 \mathrm{~Hz}$. The free-end time series and the power spectral density analysis indicate that the above frequency is a single one; therefore, no other frequency appears for the aforementioned range of values of $A$. Fig. 3 shows that for $A €[5.77,6.21]$, the attractor frequency corresponds to the frequency of the second mode, i.e. $3.50 \mathrm{~Hz}$. This implies that energy is transferred from the first to the second mode.

A very complex long time nonlinear behavior is exhibited by the self-excited beam for $A €[6.22,10]$. Fig. 3 indicates that for this range of values of $A$ the dominant frequency of the attractor corresponds to the frequency of the first mode; however, a closer look reveals a quasiperiodic behavior and the presence of multiple frequencies, as can be observed in Figs. 4 and 5, which show the free-end time series and the power spectrum for $A=10$, respectively. Power spectrum of Fig. 5 unveils the existence of several frequencies for $A=10$, being the dominant one that corresponding to the first vibration mode.

The aforementioned complex vibration behavior detected in this work for nonlinear self-excited beams is corroborated, qualitatively at least, by the fact that actual turbine and compressor blades exhibit multimodal interaction, the presence of multiple vibration frequencies and power spectral densities similar to that shown in Fig. 5 [30-32].

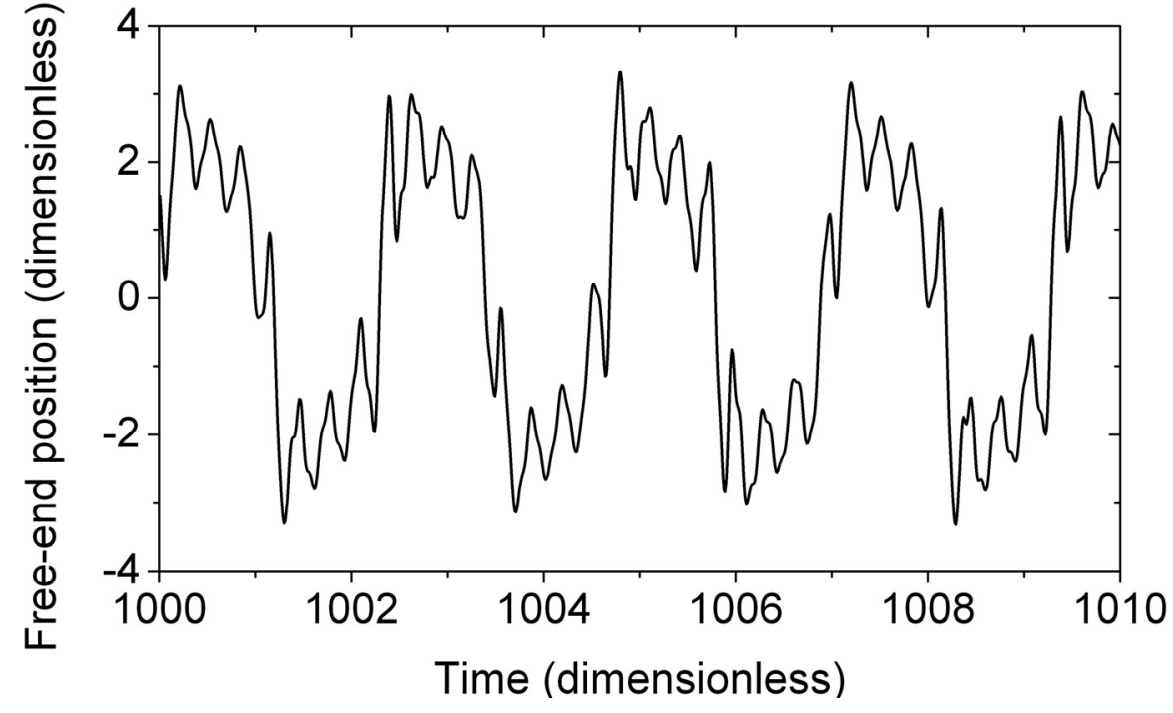

Figure 4. Time series for $A=10$. The first mode is initially excited. 


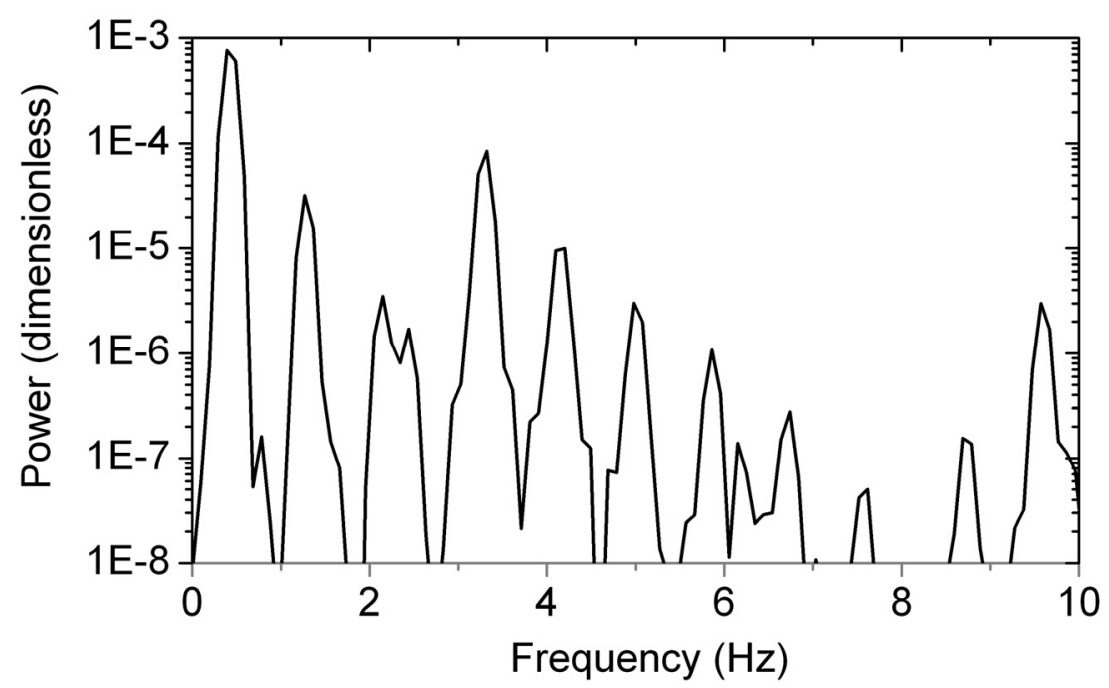

Figure 5. Power spectrum corresponding to Fig. 4.

\subsection{Energy transfer among modes}

The transfer of energy among vibration modes occurs in nonlinear beams, such as the selfexcited beam proposed in this work. The transition among modes is observed in the free-end time series of Figs. 6 and 7 for $A=3$ and $A=6$, respectively, in which just the first mode is excited. In both cases this transition occurs from a high amplitude-low frequency mode to a low amplitudehigh frequency one. For $A=3$, around 115 dimensionless time units are required to complete the transition, whereas 38 time units are required for $A=6$. This suggests that, at least for the two cases considered, dynamics is faster as the value of the self-exciting constant is increased. Experimental evidence of the energy transfer among modes, deduced from the time series of Figs. 6 and 7, has been reported in beams with external excitation [23,33].

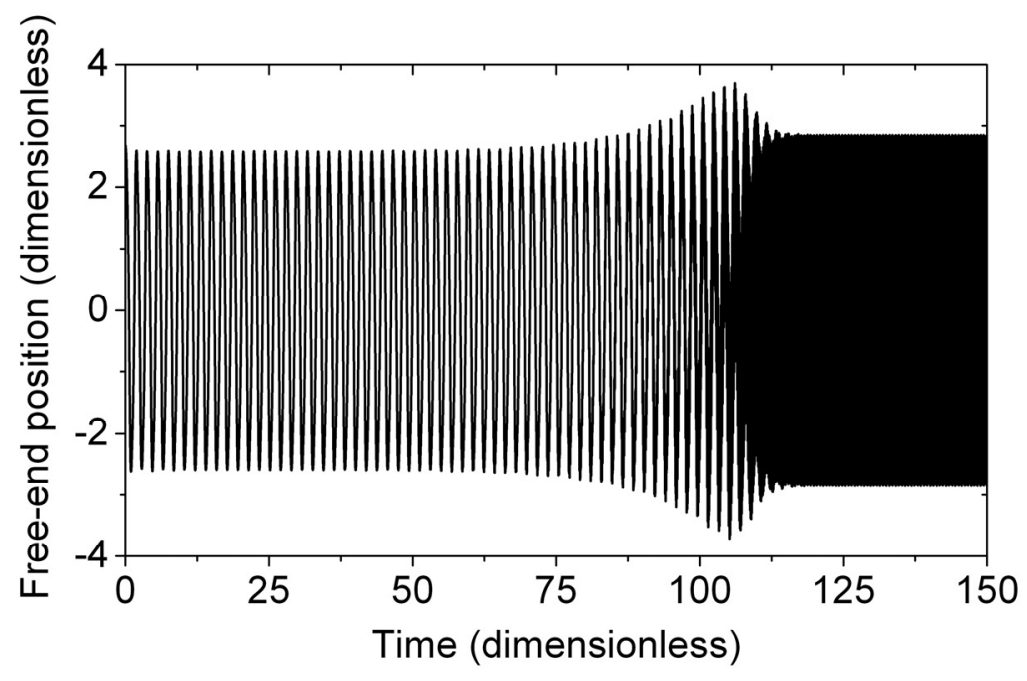

Figure 6. Transition among modes for $A=3$. The first mode is initially excited. 


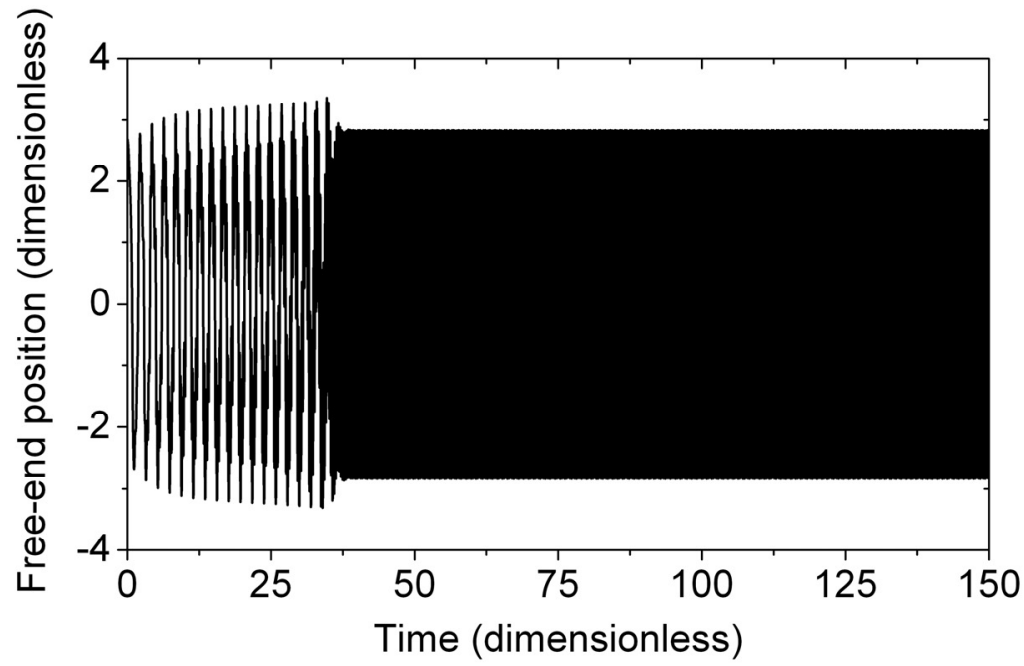

Figure 7. Transition among modes for $A=6$. The first mode is initially excited.

\section{Conclusions}

A model of a clampled-free elastic beam with a directly added van der Pol self-exciting term to simulate the vibration behavior of a turbine blade is proposed. Regions of linear and nonlinear vibration behavior of the beam are found in terms of values of the self-exciting constant. In the nonlinear region, multiple frequencies in the long term vibration behavior are detected. Transfer of energy from a low frequency mode to a high frequency one is found. The dynamics of the above transition is faster as the value of the self-exciting constant is increased.

From a qualitative point of view, the agreement between the obtained results and experimental results reported in the literature for actual beams and turbine blades is good; therefore, it can be concluded that the proposed model is potentially useful for fatigue studies and vibration analysis of blades in turbomachinery.

\section{References}

[1] Y.L. Lau, R.C.K. Leung, and R.M.C. So. Vortexinduced vibration effect on fatigue life estimate of turbine blades. Journal of Sound and Vibration, Vol. 307, 2006, pp. 698-719.
[2] Z. Mazur, R. Garcia-Illescas, J. Aguirre-Romano, and N. Perez-Rodriguez. Steam turbine blade failure analysis. Engineering Failure Analysis, Vol. 15, 2008, pp. 129-141.

[3] P. Beauseroy and R. Lengelle. Nonintrusive turbomachine blade vibration measurement system. Mechanical Systems and Signal Processing, Vol. 21, 2007, pp. 1717-1738.

[4] Y.Y. Jiang, S. Yoshimura, R. Imai, H. Katsura, T. Yoshida, and C. Kato. Quantitative evaluation of flowinduced structural vibration and noise in turbomachinery by full-scale weakly coupled simulation. Journal of Fluids and Structures, Vol. 23, 2007, pp. 531-544.

[5] C.G. Rodriguez, E. Egusquiza, and I.F. Santos. Frequencies in the vibration induced by the rotor stator interaction in a centrifugal pump turbine. ASME Journal of Fluids Engineering, Vol. 129, 2007, pp. 1428-1435.

[6] R. Violette, E. de Langre, and J. Szydlowsky. Computation of vortex-induced vibrations of long structures using a wake oscillator model: Comparison with DNS and experiments. Computers and Structures, Vol. 85, 2007, pp. 1134-1141.

[7] K.B. Skaugset and C.M. Larsen. Direct numerical simulation and experimental investigation on suppression of vortex induced vibrations of circular cylinders by radial water jets. Flow, Turbulence and Combustion, Vol. 71, 2003, pp. 35-59.

[8] E. Guilmineau and P. Queutey. Numerical simulation of vortex-induced vibration of a circular cylinder with low 
mass-damping in a turbulent flow. Journal of Fluid and Structures, Vol. 19, 2004, pp. 449-466.

[9] G.S. Pisarenko and Y.S. Vorobev. Issues of simulation of turbomachine blade vibration. Strength of Materials, Vol. 32, 2000, pp. 487-489.

[10] J. Li, S.T. Lie, and Z. Cen. Numerical analysis of dynamic behavior of stream turbine blade group. Finite Elements in Analysis and Design, Vol. 35, 2000, pp. 337-348.

[11] G. Dimitriadis, I.B. Carrington, J.R. Wright, and J.E. Copper. Blade-tip timming measurement of synchronous vibrations of rotating bladed assemblies. Mechanical Systems and Signal Processing, Vol. 16, 2002, pp. 599622.

[12] S. Kumar, N. Roy, and R. Ganguli. Monitoring low cycle fatigue damage in turbine blade using vibration characteristics. Mechanical Systems and Signal Processing, Vol. 21, 2007, pp. 480-501.

[13] M.A. Barron and M. Sen. Synchronization of coupled self-excited elastic beams. Journal of Sound and Vibration, Vol. 324, 2009, pp. 209-220.

[14] P. Bisegna and G. Caruso. Optimization of a assive vibration control scheme acting on a bladed rotor using a homogenized model. Structural and Multidisciplinary Optimization, Vol. 39, 2009, pp. 625-636.

[15] J.S. Rao and A. Saldanha. Turbomachine blade damping. Journal of Sound and Vibration, Vol. 262, 2003, pp. 731-738.

[16] R.D. Gabbai and H. Benaroya. An overview of modeling and experiments of vortex-induced vibration of circular cylinders. Journal of Sound and Vibration, Vol. 282, 2005, pp. 575-616.

[17] R.E.D. Bishop and A.Y. Hassan. The lift and drag forces on a circular cylinder in a flowing fluid. Proceedings of the Royal Society Series, Vol. A277, 1963, pp. 32-50.

[18] Y.S. Lee, A.F. Vakakis, L.A. Bergman, and D.M. McFarland. Suppression of limit cycle oscillations in the van der Pol oscillator by means of passive non-linear energy sinks. Structural Control and Health Monitoring, Vol. 13, 2006, pp. 41-75.

[19] R.T. Hartlen and I.G. Currie. Lift-oscillator model of vortex induced vibration. Journal of the Engineering Mechanics, Vol. 96, 1970, pp. 577-591.
[20] C.H.K. Williamson and R. Govardhan. A brief review of recent results in vortex-induced vibrations. Journal of Wind Engineering, Vol. 96, 2008, pp. 713-735.

[21] D. Lucor and M.S. Triantafyllou. Parametric study of a two degree-of-freedom cylinder subject to vortexinduced vibrations. Journal of Fluid and Structures, Vol. 24, 2008, pp. 1284-1293.

[22] M.L. Facchinetti, E. de Langre, and F. Biolley. Coupling of structures and wake oscillators in vortexinduced vibrations. Journal of Fluids and Structures, Vol. 19, 2004, pp. 123-140.

[23] A.H. Nayfeh. Nonlinear Interactions. Wiley, New York, NY, 2000.

[24] K.L. Graff. Wave Motion in Elastic Solids. Oxford University Press, London, UK, 1975.

[25] B. van der Pol and J. van der Mark. The heartbeat considered as a relaxation oscillation, and an electrical model of the heart. Philosophical Magazine, Vol. 6, 1928 , pp. $763-775$.

[26] J. Grasman, F. Verhulst, and S. Shih. The Lyapunov exponents of the van der Pol oscillator. Mathematical Methods in Applied Sciences, Vol. 28, 2005, pp. 11311139.

[27] D.W. Storti and R.H. Rand. Dynamics of two strongly coupled relaxation oscillators. SIAM Journal on Applied Mathematics, Vol. 46, 1986, pp. 56-67.

[28] J.S. Hesthaven, S. Gottlieb, and D. Gottlieb. Spectral Methods for Time-Dependent Problems. Cambridge University Press, Cambridge, UK, 2007.

[29] A.N. Yanmeni, R. Tchoukuegno, and P. Woafo. Non-linear dynamics of an elastic beam under moving loads. Journal of Sound and Vibration, Vol. 273, 2004, pp. 1101-1108.

[30] M. Zielinski and G. Ziller. Noncontact vibration measurements on compressor rotor blades. Measurement Science and Technology, Vol. 11, 2000, pp. 847-856.

[31] M.L.J. Verhees. Experimental Modal Analysis of a Turbine Blade. Traineship Report, Technische Universiteit Eindhoven, Eindhoven, The Netherlands, 2004.

[32] E.D. Cohen. Vibration Detection in Turbomachinery Using Non-contacting Sensors. Master's Thesis, Massachusetts Institute of Technology, Cambridge, MA, 2006. 
[33] P. Malatkar and A.H. Nayfeh. On the transfer of energy between widely spaced modes in structures. Nonlinear Dynamics, Vol. 31, 2003, pp. 225-242.

\section{Acknowledgments}

This work was partially done while the author was on sabbatical leave from the Universidad Autónoma Metropolitana-Azcapotzalco (UAMA) and visiting the University of Notre Dame (UND), Notre Dame, IN, USA. He gratefully acknowledges both the financial support from the Programa de Apoyo a Estancias Sabáticas de Investigación of the UAMA and the guidance of Professor Mihir Sen from the Department of Aerospace and Mechanical Engineering of UND. 


\section{Author's Biography}

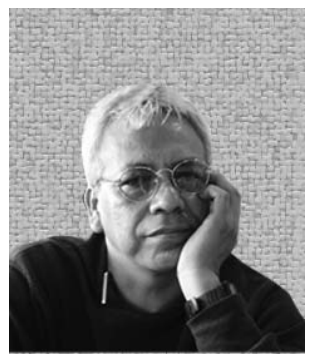

\section{Miguel Ángel BARRÓN-MEZA}

Chemical-Metallurgical Engineer from Universidad Nacional Autónoma de México. Master of Science in chemical engineering from Universidad Autónoma Metropolitana Iztapalapa, Mexico. PhD in materials science from Instituto Politécnico Nacional, Mexico. Posdoctoral fellow at Universidad Politécnica de Cataluña, Barcelona, Spain. Sabbatical fellow at University of Notre Dame, Notre Dame, IN, USA. Member of Sistema Nacional de Investigadores (National System of Researchers), Mexico. Currently, professor at Departamento de Materiales of Universidad Autónoma Metropolitana Azcapotzalco, Mexico. 